\title{
UNEIX, EL SISTEMA DE INFORMACIÓN INTERUNIVERSITARIO DE CATALUNYA
}

\section{Joan Bravo-Pijoan}

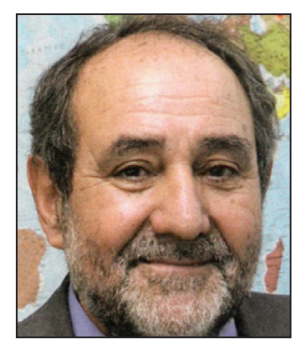

Joan Bravo-Pijoan, licenciado en economía por la Universidad de Barcelona, y máster en gestión pública por Esade, ha sido hasta 2012 secretario del Consejo de dirección de la Agencia de Calidad Universitaria de Catalunya (AQU Catalunya), y coordinador de sistemas de información del Comissionat per a Universitats i Recerca de la Generalitat de Catalunya-donde ha sido el impulsor y coordinador del sistema Uneix que hoy presenta en este artículo-. Anteriormente fue gerente del Institut d'Estadística de Catalunya (Idescat), y de su antecesor el Consorci d'Informació i Documentació de Catalunya (CIDC). En 1988 fue fundador y primer presidente de la Federación Española de Sociedades de Archivística, Biblioteconomía, Documentación y Museística (Fesabid). http://orcid.org/0000-0002-7326-4360

jbravopijoan@gmail.com

\section{Resumen}

Se describe el sistema de información Uneix, que integra los datos de gestión de doce universidades de Catalunya (7 públicas y 5 privadas), y que permite hacer un seguimiento de sus necesidades, medios y resultados: profesorado, equipos, estudios, estudiantes, mercado de trabajo para recién titulados, costes, etc. El sistema descansa sobre tres elementos: cooperación, fiabilidad de los datos -que son estrictamente revisados antes de ser introducidos- y adaptación a las necesidades de una amplia tipología de usuarios.

\section{Palabras clave}

Universidades, Aseguramiento de la calidad, Sistemas de información, Gestión, Indicadores, Costes, Rendimiento, Información para la gestión, Benchmarking, Cooperación.

\section{Title: Uneix, the interuniversity information system of Catalonia}

\begin{abstract}
Uneix, the information system that integrates the management data of twelve Catalonian universities, is described. It tracks needs, resources and results in numerous categories: faculty, staff, programs of study, students, job market for recent graduates, costs, etc. The system is based on three design elements: cooperation, data reliability (with strict quality control), and wealth of information that fits the needs of many user types.
\end{abstract}

\section{Keywords}

Universities, Quality assurance, Information systems, Management, Indicators, Costs, Performance, Dashboards, Benchmarking, Cooperation.

Bravo-Pijoan, Joan. "Uneix, el sistema de información interuniversitario de Catalunya". El profesional de la información, julio-agosto, v. 22, n. 4, pp. 363-368.

\section{Introducción}

La Declaración de Bolonia de 1999 para poner en marcha el Espacio Europeo de Educación Superior (EEES) impulsó que fueran más transparentes y compatibles los sistemas de educación superior, y que se pudiera evaluar su calidad tanto a nivel institucional como estatal. En la Conferencia de Ministros de Educación de Berlín (2003) los asistentes se comprometieron a garantizar la calidad en base a criterios y metodologías compartidos, y en la reunión de Bergen (2005) los ministros adoptaron los Criterios y directrices para la garantía de calidad en el Espacio Europeo de Edu- cación Superior ${ }^{1}$ desarrollados por la European Network for Quality Assurance in Higher Education (ENQA) con la colaboración de la European University Association (EUA), las National Unions of Students in Europe (ESIB) y la European Association of Institutions in Higher Education (Eurashe).

En el apartado 1.6 se dice en concreto: "Sistemas de información: Las instituciones deben garantizar que recopilan, analizan y utilizan información pertinente para la gestión eficaz de sus programas de estudio y otras actividades".

Esta información debe cubrir al menos los siguientes aspectos: 
- Progresión y tasas de éxito de los estudiantes

- Empleabilidad de los titulados

- Satisfacción de los estudiantes con sus programas

- Eficacia de los docentes

- Perfil de los estudiantes

- Recursos de aprendizaje disponibles y sus costes

- Indicadores de rendimiento

A su vez el apartado 1.7 establece lo siguiente: "Información pública: las instituciones deben publicar con regularidad información actualizada, imparcial y objetiva, tanto cuantitativa como cualitativa, sobre los programas y títulos que ofrecen".

Posteriormente el Parlamento Europeo y el Consejo de la Unión Europea aprobaron la recomendación 2006/143/CE instando a su aplicación y que en gran parte se fundamentaba en la Recomendación 98/561/CE $E^{3}$ de 24 de septiembre de 1998 , en la que se señalaba a los estados miembros que debían crear sistemas transparentes de evaluación y seguimiento de la calidad para poder rendir cuentas e informar al público.

En coherencia con las líneas de pensamiento renovador que dieron lugar a estas declaraciones oficiales, las universidades públicas de Catalunya, la Secretaría de Universidades e Investigación y AQU Catalunya habían iniciado ya en 1998 los trabajos para constituir en un marco de cooperación el sistema interuniversitario de información estadística: Uneix.

\section{El sistema}

Uneix (acrónimo que en catalán significa "une") se orientó a cuatro objetivos:

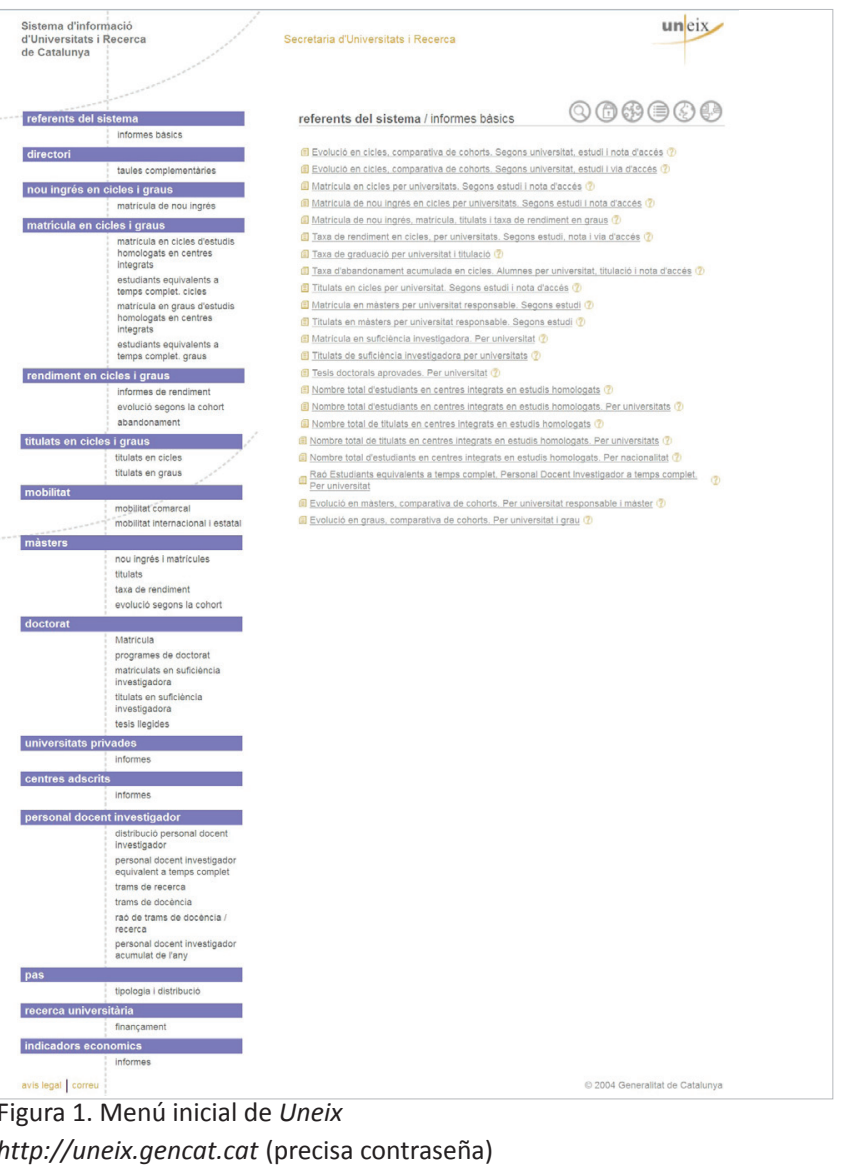

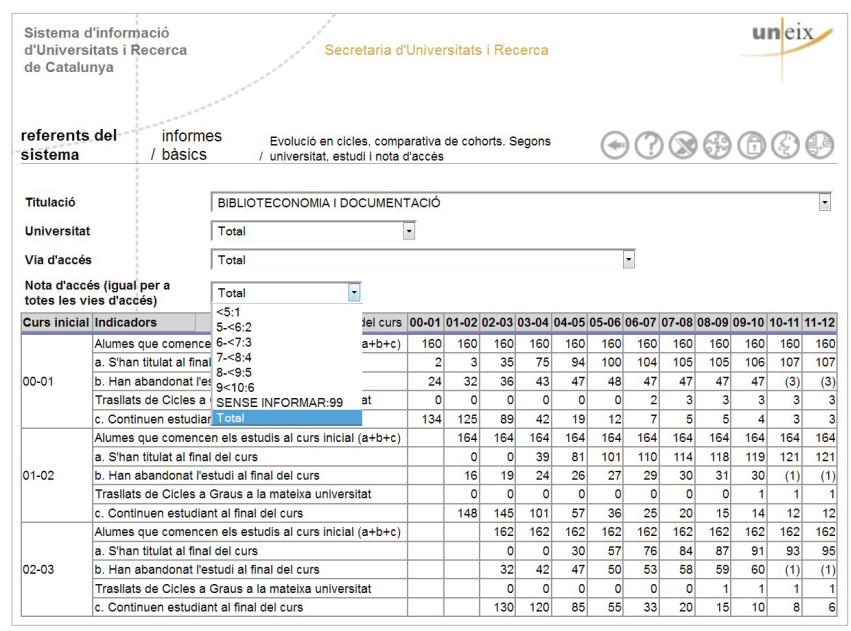

Figura 2. Estadísticas de los estudios de ByD de la UB. Una "cohorte" es el conjunto de alumnos que ingresan en el estudio en un mismo año académico.

- Contribuir al mejor conocimiento interno de cada universidad y de su posición en el conjunto del sistema universitario.

- Aprovechar el potencial pedagógico de la información para impulsar los procesos de mejora de la docencia y de la investigación.

- Incrementar la eficiencia en el uso de recursos.

- Profundizar en la exigencia democrática de transparencia y rendimiento de cuentas a la sociedad.

Tal como se puede ver en el menú inicial de la aplicación (figura 1) los datos ofrecidos por Uneix permiten alcanzar dichos objetivos.

Por ejemplo, en la figura 2 se muestra un informe donde se analizan los procesos de titulación o abandono en una titulación o estudios determinados en cada universidad que los imparte, o en el conjunto del sistema, segmentado por aspectos que inciden en el rendimiento académico como la vía y la nota de acceso de sus alumnos. El ejemplo corresponde a la titulación de biblioteconomía y documentación en la Universitat de Barcelona.

\section{Tres elementos clave}

\subsection{Cooperación}

Los datos que integran Uneix proceden de varias instituciones que, siendo autónomas, gestionan su información de acuerdo con su cultura organizativa y sus propias necesidades, y producen información con diferentes criterios y formatos. Como es esencial garantizar la homogeneidad y comparabilidad, fue necesario acordar definiciones únicas y aceptables por todos los integrantes. Para ello se crearon comisiones para analizar con detalle las definiciones, elaborar glosarios de los datos y consensuar los métodos de cálculo de los indicadores en los ámbitos de la docencia, la investigación y la economía (figura 3).

Una vez acordada en común la metodologia, las universidades tuvieron que adaptarse internamente a los nuevos criterios y procedimientos. En este sentido, es destacable que Uneix haya tenido el privilegio de disfrutar de una voluntad clara por parte de todas las instituciones de trabajar 


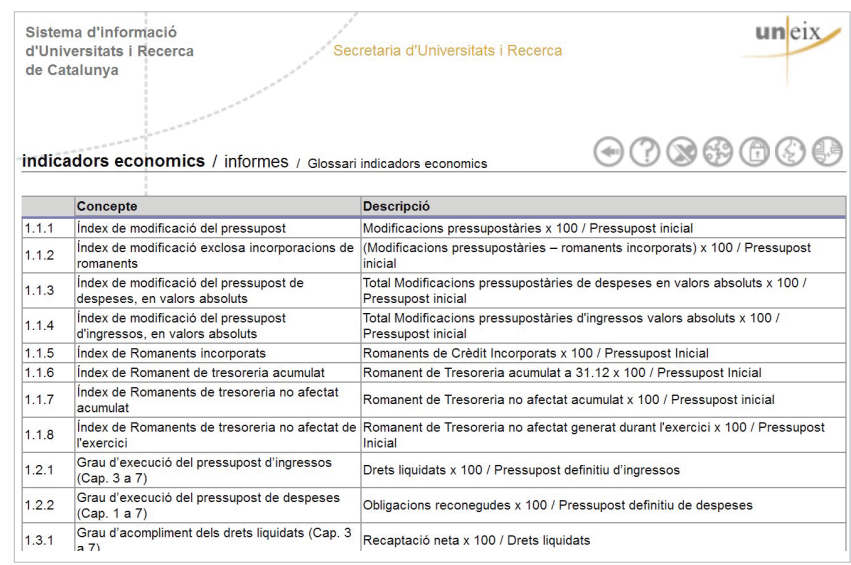

Figura 3. Ejemplo de glosario de indicadores económicos.

conjuntamente y mejorar la información disponible tanto en beneficio propio como del conjunto.

\subsection{Fiabilidad}

Un segundo aspecto crucial a considerar fue el de la validación de la información, pues el sistema no puede ofrecer datos erróneos, incompletos o en unidades no comparables. De ello depende la credibilidad de los resultados.

El proceso de validación permite detectar errores de formato, integridad, unicidad, funcionales o de contenido. Si se detecta alguna incorrección el fichero se devuelve, hasta que una vez corregido se pueda integrar en el sistema.

\subsection{Flexibilidad}

Un sistema de estas características requiere una permanente adaptación a los cambios y a las nuevas necesidades. En primer lugar, las modificaciones en las normativas inciden en las definiciones y en los criterios de codificación: nuevas titulaciones, evolución en el tipo de convocatorias de ayudas a la investigación... También cambios organizativos: división o integración de departamentos, nuevas categorías de profesorado o de investigadores...

En segundo lugar, a causa de la diversidad de usuarios: rectores y equipos de gobierno de las instituciones, respon-

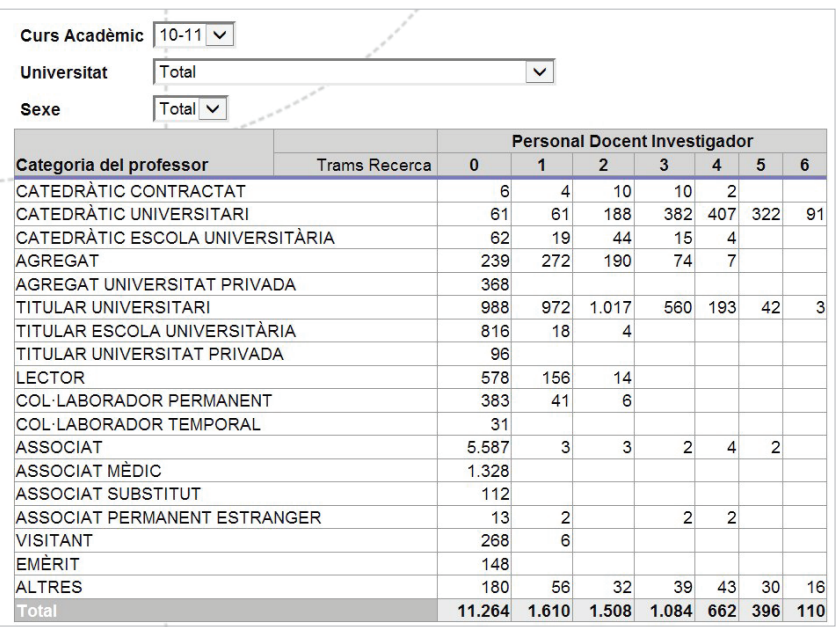

Figura 4. Sexenios o tramos de investigación del personal docente investigador. El informe permite seguir la evolución anual comparando universidades, categorías de profesorado y sexo.

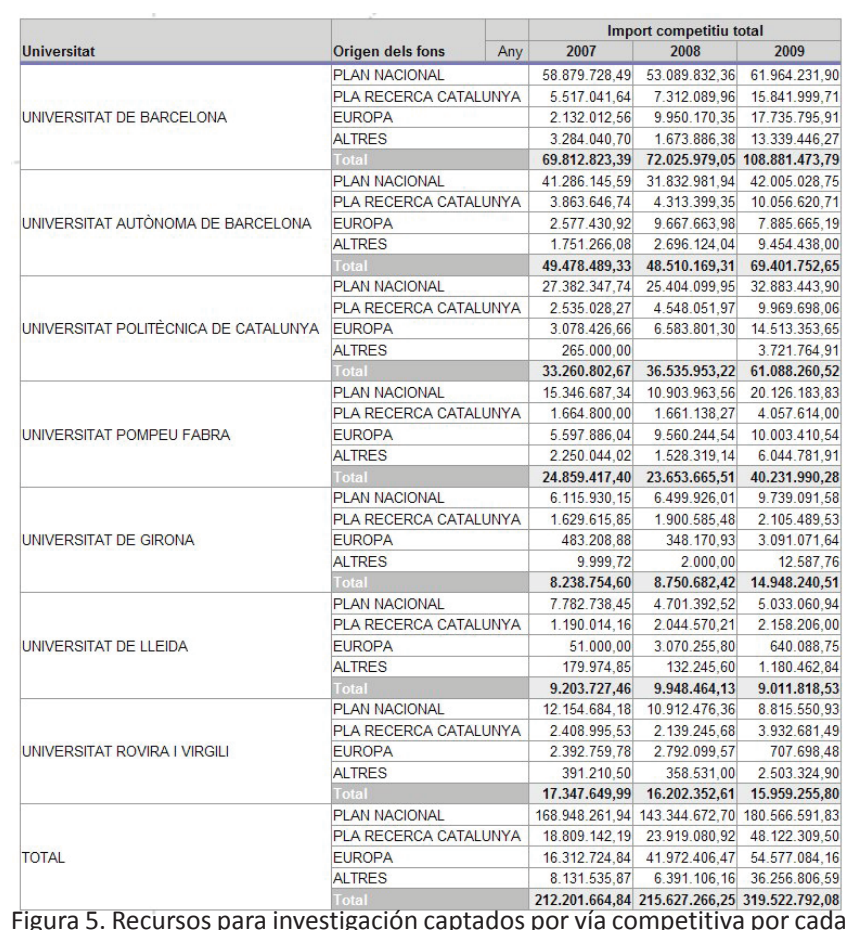

Figura 5. Recursos para investigación captados por vía competitiva por cada universidad y por fuente de financiación.

sables de la administración, profesores e investigadores, técnicos y gestores, estudiantes y sus familias, medios de comunicación..., es necesario hacer un esfuerzo específico de adaptación a los modelos de análisis de los datos.

Por último, tal como se indica en el apartado siguiente, la información disponible se ha ido ampliando como también lo han hecho las necesidades de información para el análisis, la planificación y la toma de decisiones en las diferentes áreas de gestión: personal, financiación, programación de estudios, evaluación de la calidad, investigación (figura 4), organización...

\section{Evolución de Uneix}

Los trabajos de Uneix se han diversificado en tres líneas complementarias:

- Ampliación de contenidos

- Web Winddat

- Apoyo para la creación del sistema de información universitaria de España.

\subsection{Contenidos}

Uneix se ha desarrollado por fases: docencia, investigación universitaria, centros de investigación de la salud, ampliación al conjunto de centros de investigación públicos de Catalunya, e indicadores presupuestarios y financieros (figuras 5 y 6$)$.

\subsection{La web Winddat}

La Web de Indicadores Docentes para el Desarrollo y Análisis de las Titulaciones (Winddat) se creó con el fin de hacer más asequible la información disponible en Uneix y es de uso público:

http://winddat.aqu.cat

Permite hacer el seguimiento de las titulaciones oficiales y 


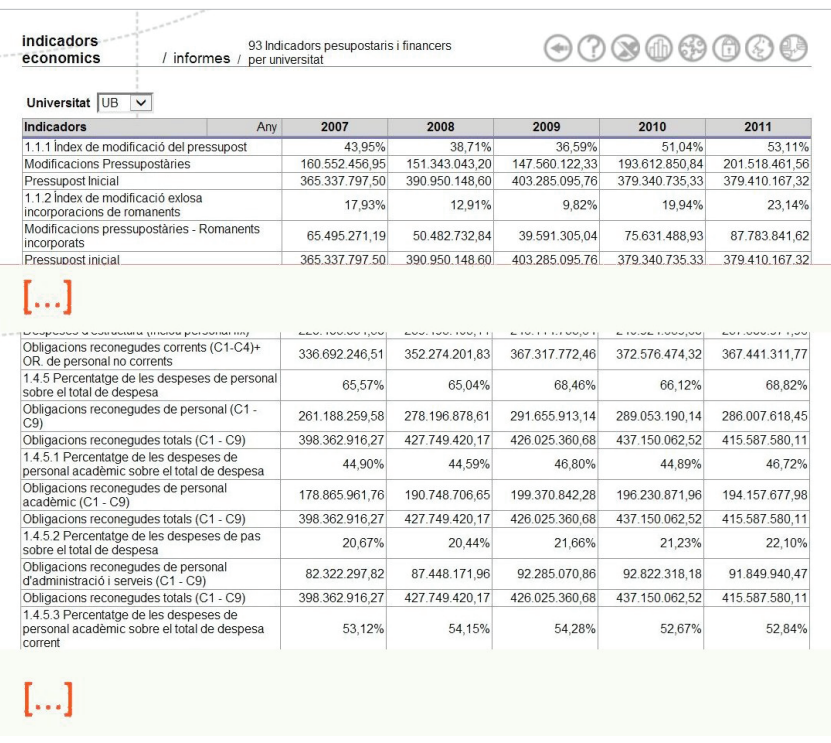

Figura 6. Porcentaje del gasto de personal sobre el total (1.4.5), diferenciando personal académico (1.4.5.1) y personal de administración y servicios (pas) (1.4.5.2), en las universidades públicas.

orientar a los estudiantes, tanto los que quieren acceder a la universidad como los que ya están cursando sus estudios, proporcionándoles datos sobre los resultados académicos y la inserción laboral en las titulaciones de su interés (figuras $7,8$ y 9$)$.

Se ha desarrollado en el marco de la Agència per a la Qualitat del Sistema Universitari de Catalunya ( $A Q U)$, en colaboración con la Universitat Autònoma de Barcelona, con el apoyo de la Secretaría de Universidades e Investigación y del conjunto de las universidades.

La información se estructura por niveles de agregación con el fin de navegar desde una visión global del sistema universitario a cada una de las universidades, a sus centros, a los distintos ámbitos temáticos y finalmente a la titulación.

\subsection{Apoyo a la creación de un sistema estatal de in- formación universitaria}

En 2007 un informe sobre la financiación universitaria en España elaborado por una comisión creada en el marco del Consejo de Coordinación Universitaria y publicado por el Ministerio de Educación (Consejo..., 2007) se refería a "las dificultades que existen para que la cultura de rendición de cuentas arraigue en las instituciones universitarias". Como se constataba entonces, evolucionar desde actitudes $o$ inercias que favorecen la opacidad a otras de impulso de la transparencia, supone un cambio cultural no exento de dificultades.

En Catalunya se habían dado pasos significativos para superar esa situación y ello, junto con la necesidad de disponer de más y mejor información sobre las universidades españolas, propició un acuerdo entre el Ministerio de Educación (en la etapa del ministro Ángel Gabilondo, 2009-2011) y la Generalitat de Catalunya. El acuerdo se firmó el 7 de julio del 2010 con el título "Convenio de colaboración entre el Ministerio de Educación y la Administración de la Generalitat de Catalunya para hacer extensivo un sistema de indicadores al conjunto del sistema universitario español en el marco del espacio Europeo de Educación Superior". Su objetivo era:

"Incorporar la experiencia avanzada de cooperación interuniversitaria en el marco del sistema Uneix de información universitario de Catalunya basado en la homogenización de las definiciones y métodos de cálculo, así como de los procesos de validación de las diversas fuentes administrativas de las universidades con el fin de garantizar el rigor en la elaboración de indicadores fiables y comparables".

Sus cláusulas establecían que "la Consejería de universidades de la Generalitat de Catalunya pondrá a disposición del Ministerio y de las comunidades autónomas las fases de desarrollo de Uneix y los procedimientos técnicos implementados".

En el convenio se explicitaban los contenidos de la colaboración y las fases para la creación del sistema estatal. Así se incluía:

En relación con el proceso de creación del sistema:

- Diseño de registros

- Definición homogénea de conceptos

- Modelo de datos y de diseño de las tablas de datos

- Campos origen y campos derivados

- Mapas de interrelaciones

- Metodología de cálculo

- Criterios de validación

- Tipología de filtros de validación

- Procesos de implementación

- Tipología de resultados

En relación con el modelo de interrelación con las universidades:

- Planteamiento de la fase previa de información y motivación

- Cooperación

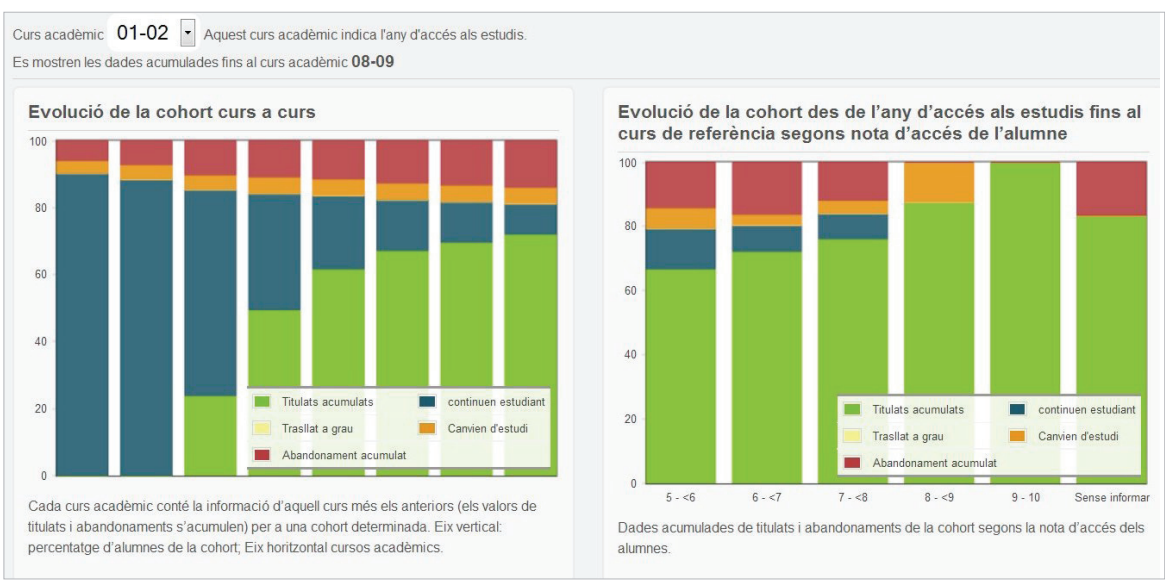

Figura 7. Situación de los estudiantes de biblioteconomía y documentación una vez transcurridos 8 años desde el inicio de sus estudios, indicando titulados, abandonos, cambios de estudio, estudiantes que continúan estudiando y trasladados al grado (EEES) 


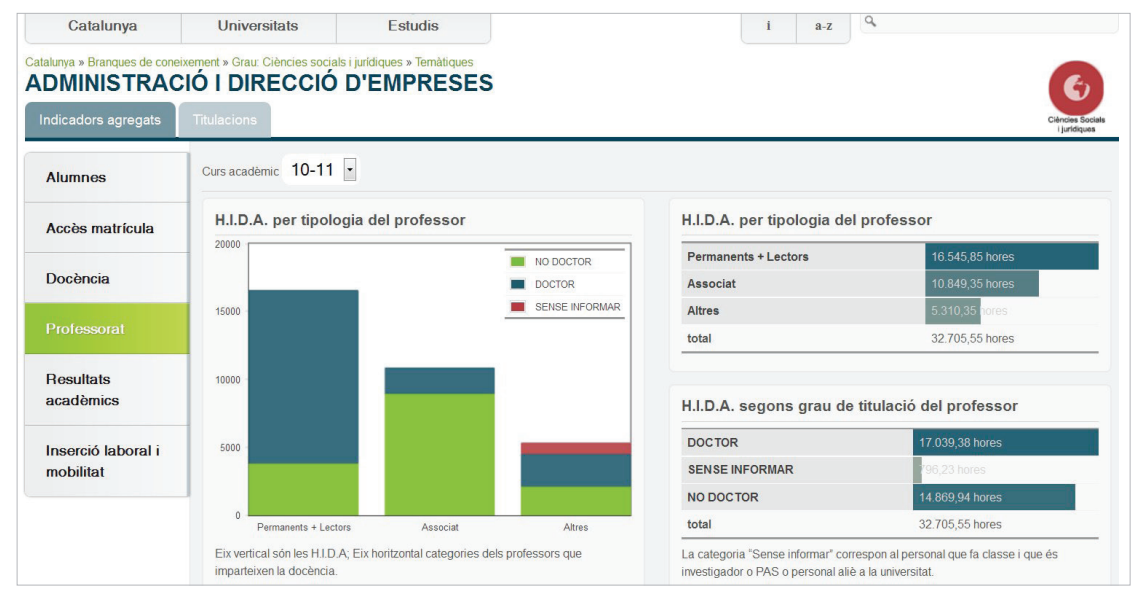

Figura 8. Horas de docencia impartidas en aula (HIDA) en los estudios de administración y dirección de empresas, según el tipo de profesor.

- Precarga

- Validación y corrección de errores

- Carga

- Análisis de resultados

- Sistema de acceso

En cuanto a las fases de elaboración de indicadores:
1. Básicos
2. Rendimiento académico
3. Recursos humanos
4. Movilidad
5. Investigación y desarrollo
6. Costes económicos
7. Inserción Laboral

Asimismo, para llevar a cabo la colaboración se estableció que "desde la Consejería de Universidades se prestaría la formación y el asesoramiento técnico necesarios para la implantación del sistema".

Con esta información, el trabajo desarrollado por el equipo técnico del Ministerio y la cooperación de las comunidades autónomas, se creó el Sistema Integrado de Información Universitaria (SIIU) destinado a dar respuesta a la lógica voluntad de cooperación entre las administraciones y al deseo de ampliar la visión sobre la situación de las universidades en el ámbito español.

En definitiva, se ha tratado de contribuir a la mejora del conocimiento del sistema universitario, de una forma que sea al mismo tiempo comprensiva y enriquecedora, funda-

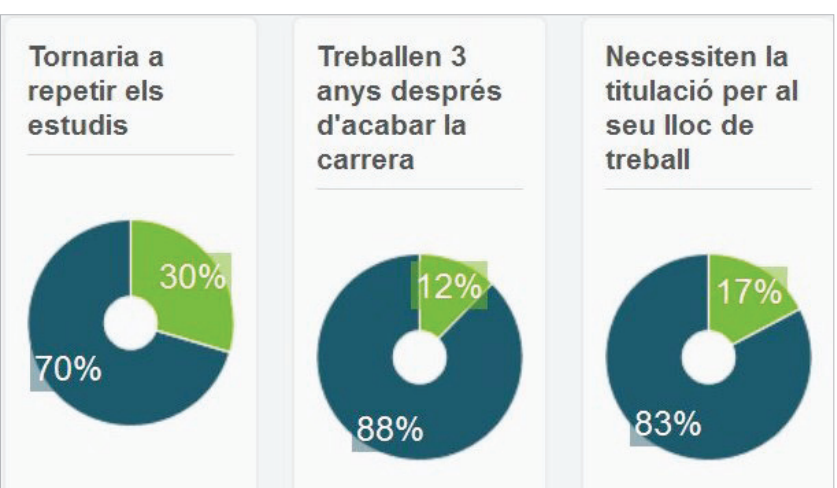

Figura 9. Situación laboral a los tres años de finalización de los estudios de química (Sí en azul oscuro, No en verde). mentada en análisis compartidos sobre una base empírica, fiable, metodológicamente homogénea y consensuada.

\section{Notas}

1. El documento original se titula Standards and guidelines for quality assurance in the European Higher Education Area. La Aneca lo tradujo al español y está disponible en:

http://www.enqa.eu/files/ESG\%20version\%20ESP.pdf

2. Recomendación del Parlamento Europeo y del Consejo de 15 de febrero de 2006 sobre una mayor cooperación europea en la garantía de la calidad de la enseñanza superior. http://eur-lex.europa.eu/LexUriServ/site/es/oj/2006/I_064/ I_06420060304es00600062.pdf

3. Recomendación 561/98/CE del Consejo, de 24 de septiembre de 1998, sobre la cooperación europea para la garantía de la calidad en la enseñanza superior [Diario oficial $L$ 270 de 7.10.1998].

http://europa.eu/legis/ation_summaries/education_training_youth/lifelong_learning/c11038_es.htm

4. En informática, un datawarehouse o almacén de datos es una base de datos pensada para analizar datos y obtener informes de situación y tendencias. Se trata de un depósito central de datos que proceden de varias fuentes, generalmente dispares.

\section{Bibliografía relacionada}

Barak, Robert J.; Kniker, Charles R. (2002). “New directions for higher education (special issue: Using benchmarking to inform practice in higher education), v. 2002, n. 118, pp. 93102.

http://dx.doi.org/10.1002/he.58

Bartoš, Vojtěch (2008). "Integrated performance measurement systems model". Ekonomika a management, v. 2008, n. 3.

http://www.vse.cz/polek/download.php?jnl=eam\&pdf=45. $p d f$

Benito, Mónica; Romera, Rosario (2011). “Improving quality assessment of composite indicators in university rankings: a case study of French and German universities of excellence". Statistics and econometrics working papers, ws112015, Universidad Carlos III de Madrid, Departamento de Estadística y Econometría.

http://e-archivo.uc3m.es/bitstream/10016/11742/1/ ws112015.pdf

Bratti, Massimiliano; McKnight, Abigail; Naylor, Robin; Smith, Jeremy (2003). "Higher education outcomes, graduate employment and university performance indicators". The Warwick economics research paper series (Twerps), 692, University of Warwick, Department of Economics. http://www2.warwick.ac.uk/fac/soc/economics/research/ workingpapers/2008/twerp692.pdf 
Capaldi, Elizabeth D.; Abbey, Craig W. (2011). "Performance and costs in higher education: a proposal for better data". Change: The magazine of higher learning, v. 43, n. 2, pp. 8-15.

http://dx.doi.org/10.1080/00091383.2011.550250

Cenar, Iuliana (2009). "The financial performance in higher education institutions. Informational supports". Annales Universitatis Apulensis Series oeconomica, Faculty of Sciences, "1 Decembrie 1918" University, Alba Iulia, v. 1, n. 11, pp. 23.

http://oeconomica.uab.ro/upload/lucrari/1120091/23.pdf

Consejo de Coordinación Universitaria (2007). Comisión de Financiación. Financiación del Sistema Universitario Español. Madrid, 20 de abril de 2007, 122 p.

http://www.crue.org/export/sites/Crue/legis/acion/ documentos/Financiacion/Financiacion_SUE_2007.pdf

Cunha, Mariana; Rocha, Vera (2012). “On the efficiency of public higher education institutions in Portugal: An exploratory study". FEP Working papers, 468, Univ. do Porto, Fac. de Economia do Porto.

http://www.fep.up.pt/investigacao/workingpapers/wp468. $p d f$

David, Fatima; Abreu, Rute; Carreira, Francisco; Gonçalves, Sidalina (2010). "Performance indicators and corporate social responsibility: evidence from Portuguese higher education institutions". International journal of banking, accounting and finance, v. 2, n. 3, pp. 251-274.

http://www.inderscience.com/link.php?id=33500

Draper, David; Gittoes, Mark (2004). "Statistical analysis of performance indicators in UK higher education". Journal of the Royal Statistical Society, Series A, v. 167, n. 3, pp. 449474.

http://www.blackwell-synergy.com/doi/abs/10.1111/ j.1467-985X.2004.apm12.x

Franceschini, Fiorenzo; Turina, Elisa (2013). "Quality improvement and redesign of performance measurement systems: an application to the academic field". Quality \& quantity: International journal of methodology, v. 47, n. 1 , pp. 465-483.

http://hdl.handle.net/10.1007/s11135-011-9530-1

Gherghina, Rodica; Nicolae, Florina; Mocanu, Mihaela (2010). "Comparative research on the correlation of the quantum to public funding for the public institutions of higher education and the institutional performance within the European Union member states". Management \& marketing, v. 5, n. 3.

http://www.managementmarketing.ro/pdf/articole/197. $p d f$
Glass, J. Colin; McCallion, Gillian; McKillop, Donal G.; Rasaratnam, Syamarlah; Stringer, Karl S. (2006). "Implications of variant efficiency measures for policy evaluations in UK higher education". Socio-economic planning sciences, v. 40, n. 2, pp. 119-142. http://dx.doi.org/10.1016/j.seps.2004.10.004

Guthrie, James; Neumann, Ruth (2007). “Economic and non-financial performance indicators in universities". Public management review, v. 9, n. 2, pp. 231-252. http://dx.doi.org/10.1080/14719030701340390

Ilie, Anca-Gabriela; Maftei, Mihaela; Colibăşanu, OanaAntonia (2011). "Sustainable success in higher education by sharing the best practices as a result of benchmarking process". The Amfiteatru economic journal, v. 13 (special N), pp. 688-697.

http://www.amfiteatrueconomic.ro/temp/Article_1081.pdf

Johnes, Geraint (1992). "Performance indicators in higher education: a survey of recent work". Oxford review of economic policy, v. 8, n. 2, pp. 19-34.

http://hdl.handle.net/10.1080/14719030701340390

Kong, Wei-Hsin; Fu, Tsu-Tan (2012). "Assessing the performance of business colleges in Taiwan using data envelopment analysis and student based value-added performance indicators". Omega, v. 40, n. 5, pp. 541-549.

http://dx.doi.org/10.1016/j.omega.2011.10.004

Martín, Emilio (2003). "An application of the data envelopment analysis methodology in the performance assessment of the Zaragoza University Departments". Documentos de trabajo: dt2003-06, Facultad de Ciencias Económicas y Empresariales, Universidad de Zaragoza.

http://www.dteconz.unizar.es/DT2003-06.pdf

McKinnon, Kenneth R.; Walker, Suzanne H.; Davis, Dorothy V. (2000). Benchmarking: a manual for Australian universities. Department of Education, Training and Youth Affairs (Detya), Australia, ix, 167 pp. ISBN: 0642239711

McMillan, Melville L.; Datta, Debasish (1998). "The relative efficiencies of Canadian universities: A DEA perspective". Canadian public policy, v. 24, n. 4, pp. 485-511.

Ng, Ying-Chu; Li, Sung-Ko (2000). "Measuring the research performance of chinese higher education institutions: an application of data envelopment analysis". Education economics, v. 8, n. 2, pp. 139-156.

Yang, Lijing; McCall, Brian (2013). "World education finance policies and higher education access: A statistical analysis of world development indicators for 86 countries". International journal of educational development. En prensa. http://dx.doi.org/10.1016/j.ijedudev.2012.11.002 